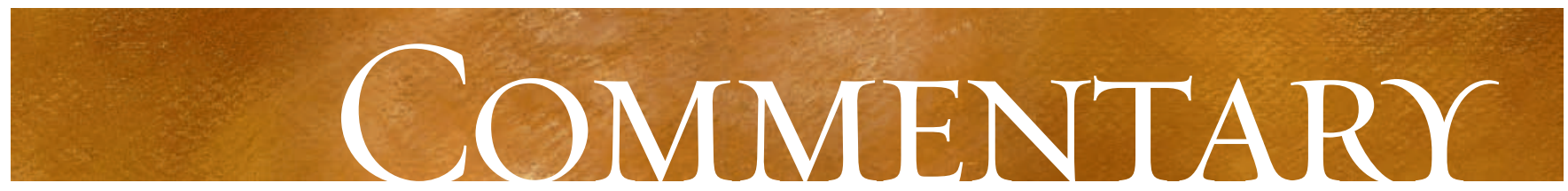

\title{
Kidney transplantation in Canada: unequal access
}

\section{Jeffrey S. Zaltzman}

$\infty \quad$ See related article page 478

$\mathrm{K}$ idney transplantation in Canada is limited by the availability of organs, not by financial constraints. Inherent in transplantation is the ethical tension of allocating a scarce resource. Every new patient placed on a transplant waiting list must compete with other patients for organs. Unlike most other medical procedures available, patients die while waiting for organ transplantation. In 2004 in Canada, 224 (6\%) patients died while on transplant waiting lists. ${ }^{1}$ Not included in these statistics are the patients never referred for transplantation and those withdrawn from the waiting list. Statistics for these patients are not available. In addition, it has become increasingly evident that kidney transplant outcomes are inferior once waiting times exceed 2 years, and especially so after 7 years. ${ }^{2}$

In this issue of CMAJ, Tonelli and coauthors examine whether Canada's geographic size influences patients' chances of kidney transplantation from deceased donors. ${ }^{3}$ They looked at 2 key factors: the geographic region in which patients resided at the time dialysis was initiated, and the distance from the patient's residence to the closest transplant centre. Surprisingly, distance from the transplant centre was not a barrier to receiving a kidney transplant. Patients who lived more than $300 \mathrm{~km}$ from a centre were just as likely as those who lived within $50 \mathrm{~km}$ to receive a kidney. However, there were large variations between the 7 provincial regions studied. For example, patients in Alberta were 3.74 times more likely ( $95 \%$ confidence interval $2.95-4.76$ ) than those in Ontario to receive a kidney transplant from a deceased donor. What must not to be overlooked is the relatively small proportion of patients who received kidney transplants from deceased and live donors during the median study period of 2.4 years: only 10.6\% and $5.8 \%$ donors respectively. Also, $46 \%$ of the patients died during that time, which illustrates the high mortality of end-stage renal disease.

One limitation of their study was the lack of information on which patients were referred for pre-transplantation evaluation and how many of them were deemed eligible and ultimately placed on the waiting list. It is well known that placement on transplant waiting lists can be quite variable. Although comparisons between provinces are not available, it is known that access to transplant waiting lists is limited by both the referring dialysis centres and the transplant centre. Garg and associates showed that, in the United States, referral of dialysis patients for transplantation was significantly lower in nonacademic, for-profit dialysis centres than in academic, not-for-profit centres. ${ }^{4}$ The hypothesis was that it was profitable for physicians to maintain their patients on dialysis. Similarly, although Canadian guidelines have been established for assessing kidney transplant candidacy, ${ }^{5}$ transplant centres probably vary in their willingness to list patients with end-stage renal disease and increasing comorbidity. The true waiting list accrual rate is unknown. It is unknown whether the number of patients referred by primary nephrologists or accepted by the transplant physician and who live in more remote areas differs from the number of those living closer to the transplant centre.

\section{Canada has no national} \section{transplant organization with coordinated organ sharing and allocation.}

As for the provincial differences, the data provided by Tonelli and colleagues suggest that dialysis patients living in Atlantic Canada, Quebec, Saskatchewan and Alberta have a decided advantage over patients in Ontario, Manitoba and British Columbia in gaining access to kidney transplantation from deceased donors. What are the reasons for this? The waiting time for a kidney from a deceased donor is a function of access to the list (referral to and acceptance by a transplant physician), the number of listed patients and the organ donation rate. Provincial rates of organ donation are readily available. In fact, there is internal consistency between the results provided by Tonelli and colleagues, since the waiting times noted in their article reflect the varying provincial rates. In 2002, the number of organ donations per million population in Canada was I3. In that same year, the rates in Alberta, Quebec and Atlantic Canada were I7.I, I7.I and I5.2 respectively, whereas in the provinces with the longest waiting times - British Columbia and Ontario - the donation rates were 7.3 and II.I respectively. ${ }^{1}$

There are 3 other important issues that need to be clarified. First, in all but 2 provinces patients can be placed on the 
waiting list for a kidney transplant from a deceased donor when their glomerular filtration rate is less than $20 \mathrm{~mL} / \mathrm{min}$. However, in British Columbia and Ontario they have to wait until they have begun dialysis treatment. This variation in practice makes the longer waiting times in British Columbia and Ontario look even worse. For example, not only do patients in Alberta who undergo dialysis have decreased waiting times for kidney transplantation, Albertans with renal failure who are not yet receiving dialysis also have access to the pool of kidneys from deceased donors. From 2000 to 2003, there were 37,23 and II pre-emptive kidney transplants from deceased donors in Quebec, Alberta and Atlantic Canada respectively. ${ }^{1}$

Second, Tonelli and colleagues report on variations in provincial waiting times for kidney transplantation. In most provinces, there is a single waiting list, and the kidneys are allocated directly to the patients. Therefore, all eligible waitlisted adult recipients in most provinces have equal access to the entire provincial donor pool. Kidneys are allocated based on provincially accepted criteria, with priority given to pediatric recipients. Ontario differs from the other provinces in that each of its 5 kidney transplant regions (London, Hamilton, Toronto, Ottawa and Kingston) has its own waiting list. Although there are provincial allocation standards agreed upon by the transplant centres, kidneys are generally allocated locally. Thus, residence location can influence waiting times in Ontario, since the regions vary in supply and demand for kidneys. The most recent data, from Apr. I through June I5, 2006, revealed that patients in Ottawa who received a kidney from a deceased donor waited on average 930 days, as compared with $\mathrm{I} 489$ days in Toronto (Trillium Gift of Life database). Therefore, although Ontario looks bad on the whole, it must be remembered that some regions in Ontario do quite well, whereas others bring down the Ontario average.

Lastly, many centres have tried to compensate for poor organ donation rates by increasing resources required for donation from living donors. This has been demonstrated in centres in Vancouver, Hamilton and Toronto. By contrast, Quebec, with one of the best rates of organ donation from deceased donors in the country, has the lowest rate from living donors. ${ }^{1}$

In summary, Tonelli and colleagues should be commended for addressing some important issues affecting access to kidney transplantation. Although their study had limitations, it appears that distance from the nearest transplant centre is not a barrier to kidney transplantation, if one assumes that access to the waiting list is not an issue. In con- trast, there is marked disparity between provinces in terms of waiting times.

Unlike the United States and member countries of Eurotransplant, Canada does not have a single national transplant organization with coordinated organ sharing and allocation. Although this would be a bold initiative, it is unclear whether such an organization would actually have an impact on the actual number of transplants being performed. Some work has been done in trying to at least consider a national sharing of kidneys for the highly sensitized recipients (those with high levels of anti-HLA antibodies), a cohort of very disadvantaged patients. ${ }^{6}$

In order to address the disparity in organ donation rates, best practices from jurisdictions with the highest donation rates need to be examined and adopted. On the positive side, some provincial organ donation agencies have begun to initiate best practice patterns and are working in greater collaboration with hospitals and intensivists in their jurisdictions, using models developed in the United States and Canada.

This article has been peer reviewed.

Jeffrey Zaltzman is with the Department of Medicine, Division of Nephrology, St. Michael's Hospital, Toronto, Ont.

Competing interests: Jeffrey Zaltzman has received consultancy fees from Novartis Pharmaceuticals Canada Inc., Wyeth Pharmaceuticals and Astellas Pharma Canada Inc.

\section{REFERENCES}

I. Canadian Institute for Health Information (CIHI). Patients who died while waiting for a transplant, Canada and provinces, summary statistics, January I to December 3I, 2004 [Table 2B]. Ottawa: CIHI; 2005. Available: http://secure.cihi.ca/cihiweb /en/downloads/CORRQ404_Table2B_C_e.pdf (accessed 2006 July 28).

2. Okechukwu CN, Lopes AA, Stack AG, et al. Impact of years of dialysis therapy on mortality risk and the characteristics of longer term dialysis survivors. Am J Kidney Dis 2002;39:533-8.

3. Tonelli M, Klarenbach S, Manns B, et al; Alberta Kidney Disease Network. Residence location and likelihood of kidney transplantation. CMAJ 2006;175(5):478-82.

4. Garg PP, Frick KD, Diener-West M, et al. Effect of the ownership of dialysis facilities on patients' survival and referral for transplantation. N Engl J Med I999;34I:I653-60.

5. Knoll G, Cockfield S, Blydt-Hansen T, et al. Canadian Society of Transplantation consensus guidelines on eligibility for kidney transplantation. $C M A J$ 2005;173(10): II8I-4.

6. Canadian Council for Donation and Transplantation. Recommendations: assessment and management of immunologic risk in transplantation. Available: www.ccdt.ca/english/recommendations/immunologic.html (accessed $2006 \mathrm{Jul} 20$ ).

Correspondence to: Dr. Jeffrey S. Zaltzman, Division of Nephrology, Department of Medicine, St. Michael's Hospital, 30 Bond St., Toronto ON M5B IW8; fax 416 867-3709; jeffrey.zaltzman@utoronto.ca

\section{TrAdition}

Since I9II, CMAJ has been committed to advancing the science and art of medicine and promoting public health. 